\title{
THEMA
}

\section{O Uso do GeoGebra para o Ensino de Cálculo Diferencial e Integral, um Mapeamento de Suas Publicações}

\section{The Use of Geogebra for Differential and Comprehensive Calculation, a Mapping of its Publications}

\author{
Ricardo Augusto de Oliveira1; William Vieira Gonçalves; Diego Piasson¹
}

\section{RESUMO}

Propomos um mapeamento dos artigos, teses e dissertações que tratam da temática "Ensino de Cálculo Diferencial e Integral (CDI) com o uso do GeoGebra", entre os anos de 2010 a 2018². Para tal, foram analisadas publicações online, advindas de cinco bases de dados científicos. Concentrou-se em reconhecer características e aspectos didático pedagógicos que envolvem o uso do GeoGebra para o ensino do CDI. O viés metodológico deste trabalho é quanti-qualitativa, e o procedimento metodológico utilizado foi a pesquisa bibliográfica no modelo "Estado da Arte". O principal resultado obtido foi a constatação de que o uso do GeoGebra é significativo, viável e importante para o ensino de CDI em sala de aula.

Palavras-chave: Ensino, GeoGebra, Cálculo Diferencial e Integral.

\begin{abstract}
We propose a mapping of the articles, theses and dissertations that deal with the theme "Teaching Differential and Integral Calculus (CDI) with the use of GeoGebra" between the years 2010 and 2018. To this end, we analyzed online publications from six scientific databases. It focused on recognizing characteristics and pedagogical didactic aspects that involve the use of GeoGebra for teaching the CDI. The methodological bias of this work is quanti-qualitative, and the methodological procedure used was the bibliographic research in the "State of Art" model. The main result obtained was the finding that the use of GeoGebra is significant, feasible and important for the teaching of CDI in the classroom.
\end{abstract}

Keywords: Teaching, GeoGebra, Differential and Integral Calculus.

\footnotetext{
${ }^{1}$ UNEMAT - Universidade do Estado de Mato Grosso, Barra do Bugres/MT - Brasil

2 A pesquisa se dá no âmbito do Projeto de Pesquisa: Tecnologias Digitais para formação inicial e continuada de professores de matemática, que se desenvolve junto a UNEMAT polo de Barra do Bugres - MT, e como parte de um Projeto de Dissertação do autor, a fim de contribuir com as pesquisas elencadas, que tem como um de seus propósitos, formular parâmetros de atividades e designer de curso para o ensino de matemática.
} 


\section{INTRODUÇÃO}

O ensino de Cálculo Diferencial e Integral, nas universidades do Brasil e do mundo, há tempos vem sendo alvo de questionamento quanto à metodologia de ensino adotada, 0 alto índice de reprovações e a dificuldades encontrada pelos alunos recém-chegados do Ensino Médio, assim como afirmam Silva (2017) e Martins Junior (2015).

Dentre as propostas encontradas na literatura para enfrentar tal situação estão as que utilizam o GeoGebra no ensino. O GeoGebra tem se mostrado um importante recurso pedagógico ao permitir: aplicar atividades investigativas (ALVES, 2016, p. 130), situações problemas (ARANGO, GAVIRIA e VALENCIA, 2015, p. 415); fazer uso de applets e sequências didáticas (CUNHA e LAUDARES, 2017), ou mesmo para explorar conceitos (SIMÕES, 2014, p. 27), (BIZELLI, BARROSO e FISCARELLI, 2001, p. 10); e realizar construções que auxiliam na resolução de situações problemas (NUMER e JUSTO, 2015, p. 01).

Neste trabalho, pretendemos motivar a realização de uma discussão sobre "O ensino de Cálculo Diferencial e Integral com o uso do software GeoGebra", apresentando um mapeamento de pesquisas, que têm como principal objetivo construir um panorama teórico prático da temática.

Ao longo do trabalho, procuramos identificar as razões apontadas pelos pesquisadores para as dificuldades no ensino de Cálculo e as propostas para seu enfrentamento. Também realizamos uma descrição de como o GeoGebra tem sido utilizado para o ensino desta disciplina (geração de applets, exploração ou explicação de conteúdos); identificamos as metodologias adotadas pelos pesquisadores (trabalhos com situações problemas, sequências didáticas, atividades direcionadas); apontamos os argumentos pelos quais o software é utilizado.

Para tanto, foram realizadas pesquisas junto às bases de dados nacionais e internacionais DIRECT SCIENCE ${ }^{3}$, PORTAL DE PERIÓDICOS CAPES ${ }^{4}$, SCIELO ${ }^{5}$, WEB OF SCIENCE ${ }^{6}$, GALE ${ }^{7}$, SCOPUS ${ }^{8}$, durante o mês de fevereiro do corrente ano. Procurou-se identificar produções que abordassem o uso do GeoGebra para ensino de Cálculo Diferencial e Integral (CDI).

A metodologia de pesquisa adotada para o trabalho foi a quanti-qualitativa, a qual, segundo Garnica e Pereira (1997), os dados quantitativos não são considerados os objetos principais, mas servem de direcionamento para a análise.

A pesquisa se caracteriza no modelo "Estado da Arte" pois busca realizar um levantamento de pesquisas, movidos pelo desafio de se conhecer o que há de publicações sobre o assunto, conforme cita Palanch (2016), onde ao se optar por este modelo de pesquisa, os pesquisadores são guiados pelo desafio de conhecer o que já têm construído e depois buscar identificar o que ainda não foi.

Para análise dos trabalhos procurou-se responder os seguintes questionamentos: A pesquisa é nacional ou internacional? Quais são seus sujeitos? A pesquisa é empírica ou teórica? Discorre sobre dificuldades no ensino de Cálculo? Sugere propostas de atividades que possam contribuir com seu

\footnotetext{
${ }^{3}$ Disponível em: <https://www.sciencedirect.com/ > acesso em: 06/02/2018.

${ }^{4}$ Disponível em: <http://portal.periodicos.capes.gov.br $>$ acesso em: 06/02/18.

${ }^{5}$ Disponível em: $<$ http://www.scielo.br/?lng=pt $>$ acesso em: 06/02/18.

${ }^{6}$ Disponível em: $<$ https://webofknowledge.com/ > acesso em: 06/02/18.

7 Disponível em:< https://www.gale.com/uk $>$ acesso: 06/02/18.

${ }^{8}$ Disponível em: < https://www.elsevier.com/americalatina/pt-br/scopus $>$ acesso em: 06/02/18.
} 
enfrentamento? No desenvolvimento do trabalho, aborda o GeoGebra para: (1) realizar explicação de um dado conteúdo; (2) explorar conceitos a partir de construções realizadas pelos sujeitos de pesquisa; e (3) objetos de aprendizagem através dos applets por ele gerado?

\section{METODOLOGIA}

A metodologia de pesquisa adotada foi a quanti-qualitativa. Segundo Garnica e Pereira (1997), o caráter quantitativo permite ao pesquisador identificar direções de análises a serem perseguidas, e servem de base para o direcionamento da análise da pesquisa.

Para Abragnando (1962), o caráter quantitativo de uma pesquisa tem a ver com o mundo das grandezas expressa por quantidades, enquanto o qualitativo, por sua vez, está relacionado às condições de atributos que distinguem uma coisa da outra, a um adjetivo de qualidade, uma condição, característica.

Ainda segundo Garnica e Pereira (1997), uma pesquisa qualitativa analisa a qualidade das informações, dos dados que estuda, o conteúdo das mesmas e não somente a quantidade, o número de informações. Permite que a pesquisa possa traduzir sensações e opiniões presentes nas informações.

A pesquisa se classifica ainda como Estado da Arte, em acordo com Palanch (2016), pois visa conhecer os dados levantados, os percursos, os resultados, enfim, identificar o que dizem as pesquisas sobre um determinado tema, e a partir destas informações, traçar novos caminhos de investigação. Ainda segundo Palanch (2016), este modelo de pesquisa visa à descrição da pesquisa, um processo de levantamento de informações acerca de um dado tema, num dado lugar e período.

[...] entendemos o mapeamento da pesquisa como um processo sistemático de levantamento e descrição de informações acerca das pesquisas produzidas sobre um campo específico de estudo, abrangendo um determinado espaço (lugar) e período de tempo. Essas informações dizem respeito aos aspectos físicos dessa produção (descrevendo onde, quando e quantos estudos foram produzidos ao longo do período e quem foram os autores e participantes dessa produção), bem como aos seus aspectos teórico-metodológicos e temáticos (FIORENTINI, PASSOS e LIMA, 2016, p. 18) apud (LIMA, BIANCHINI e GOMES, 2017, p. 2)

Assim, nos propusemos a mapear trabalhos científicos acerca do tema em questão com publicações datadas de 2010 até o presente ano.

As fontes de pesquisas escolhidas para a realização do mapeamento (DIRECT SCIENCE, CAPES, SCIELO, WEB OF SCIENCE, GALE, SCOPUS), deu-se em função do grau de importância, nacional e internacional, dos trabalhos na área de ensino nelas encontrados, e por disponibilizarem o que há de mais recentes na área de investigação deste trabalho.

A Web of Science é uma base de dados científica que conecta publicações e pesquisadores do mundo todo. Em seus registros, encontra-se mais de cem anos de conteúdo totalmente indexado, incluindo cinquenta e nove milhões de registros e backfiles que datam publicações desde 1898. Seu caráter 
multidisciplinar de publicações possibilita acesso via CAPES de resumos e pesquisas das mais diversas áreas, sendo este um importante veículo de buscas também para a área de ensino. (BRASIL, 2018) ${ }^{9}$.

Gale é uma base de dados científica que, por mais de sessenta anos, fez parcerias com bibliotecas em todo o mundo, presente em cinco continentes, cento e quarenta países, detém de um vasto catálogo de conteúdo com mais de cem disciplinas e quatro mil áreas de conhecimento. A editora é especializada em oferecer soluções de aprendizagem para universidades, escolas e instituições, na forma de artigos, o que torna suas publicações interessantes para a pesquisa na área de ensino. $(\text { BRASIl, Idem) })^{10}$.

Scopus é a maior base de dados de citações e resumo de literatura revisada por pares do mundo inteiro, contendo: revistas científicas, livros e conferências. Oferece ferramentas inteligentes para rastrear, analisar e visualizar pesquisas nas áreas de ciência, tecnologia, medicina, ciências sociais, artes e humanidades. Trata-se de uma plataforma que atende a toda comunidade acadêmica, incluindo pesquisadores, graduandos, docentes, pós-graduandos e bibliotecários. Tem como objetivo a constante ampliação e melhoria de pesquisas e trabalhos científicos, sendo este um contexto importante para a área de ensino. (BRASIL, Idem) ${ }^{11}$.

SciELO é uma biblioteca eletrônica que abrange uma coleção selecionada de periódicos científicos brasileiros. Resultado de um projeto de pesquisa da FAPESP - Fundação de Amparo à Pesquisa do Estado de São Paulo, em parceria com a BIREME - Centro Latino-Americano e do Caribe de Informação em Ciências da Saúde. Desde 2002, o projeto passou a contar com o apoio do CNPq - Conselho Nacional de Desenvolvimento Científico e Tecnológico. O objetivo da Scielo é contribuir para o avanço da pesquisa e aperfeiçoar os periódicos do país, visando a qualidade e o aumento da visibilidade nacional e internacional dos trabalhos publicados (BRASIL, Idem). ${ }^{12}$

A CAPES é o portal brasileiro de informação científica. Com este serviço, alunos, professores, pesquisadores e funcionários de cento e noventa e um instituições de ensino superior e de pesquisa em todo o país podem ter acesso à produção científica mundial atualizada. (BRASIL, Idem)

A Science Direct é uma base de dados vinculada a Editora Elsevier Brasil, assim como a Scopus criada em 1997. É uma plataforma anglo-Holandesa, que permite acesso há mais de duas mil e quinhentas revistas científicas, vinte e seis mil e-books. Ainda, são ferramentas importantes para introdução de estudos acadêmicos em fase inicial em cursos superiores, pesquisa e iniciação científica. Os títulos podem ser utilizados por professores e alunos tanto da graduação quanto da pós-graduação. (BRASIL, Idem $)^{13}$

\footnotetext{
${ }^{9}$ Disponível em:

$<$ http://www.periodicos.capes.gov.br/?option=com pnews\&component=NewsShow\&view=pnewsnewsshow\&cid=161\&m $\underline{\mathrm{n}=0}>$ acesso em 10/02/2028

${ }_{10}$ Disponível em:< http://www.cengage.com.br/blog/gale/ > acesso em: 10/02/2018

11 Disponível em:< http://www.capes.gov.br/sala-de-imprensa/noticias/8499-plataforma-de-resumos-e-citacoes-reunemais-de-60-milhoes-de-registros $>$ acesso em 10/02/2018

12 Disponível em:< http://www.capes.gov.br/bolsas/programas-especiais/parceria-capes-fapunifesp-programa-scielo > acesso em 10/02/2018

13 Disponível em:< http://www.capes.gov.br/sala-de-imprensa/noticias/8391-usuarios-do-portal-de-periodicos-tem-acessoa-40-titulos-em-portuques-do-sciencedirect > acesso em: 10/02/2018
} 
Para a seleção dos trabalhos, nas bases mencionadas, foram utilizados os descritores "GeoGebra", "Ensino" e "Cálculo Diferencial e Integral". Primeiramente, foram realizadas buscas utilizando conjuntamente os descritores "GeoGebra" e "Ensino de Cálculo Diferencial e Integral".

A Imagem 1 apresenta a busca realizada na CAPES pelo descritor "Cálculo Diferencial e Integral".

Imagem 1 - Exemplo de buscas realizadas na CAPES

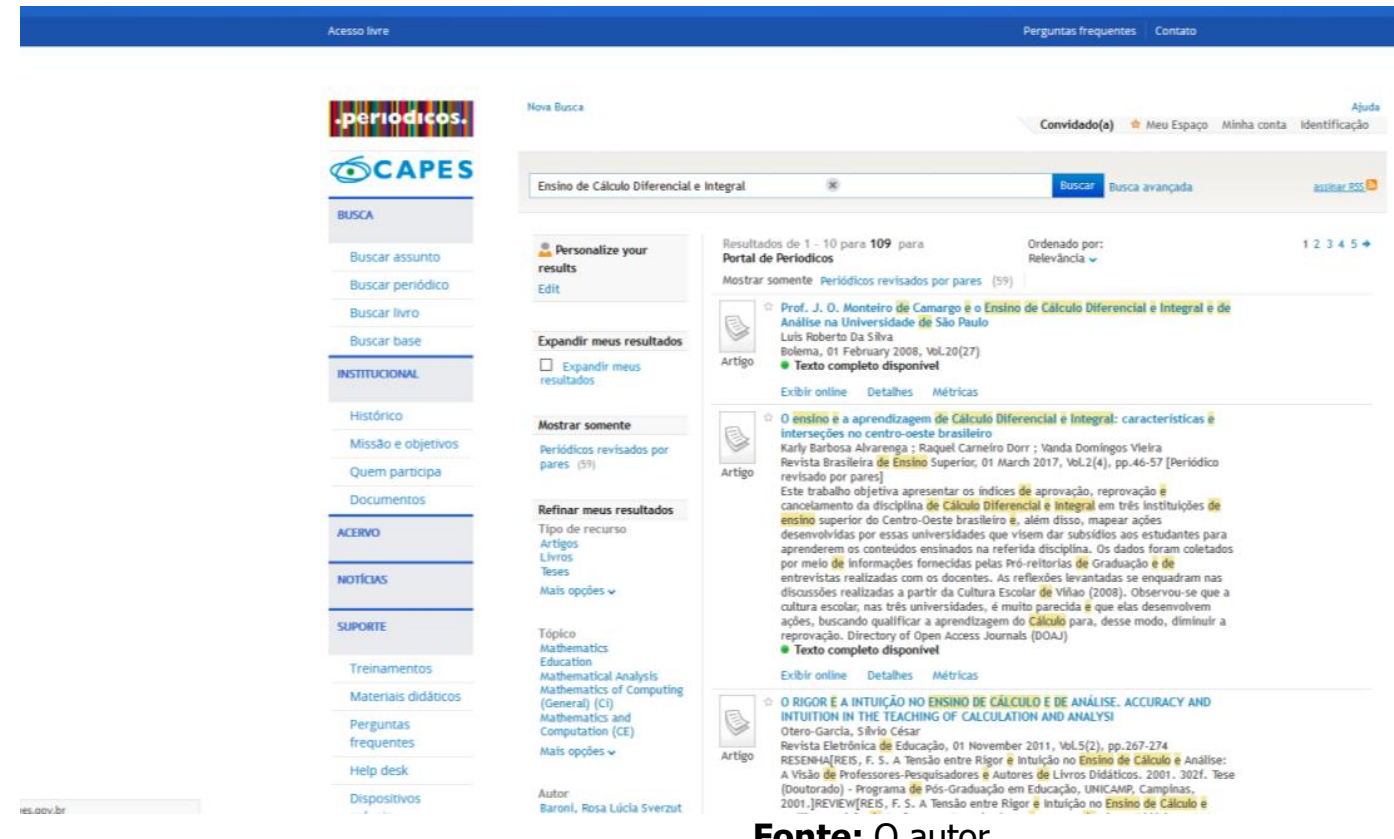

Fonte: $\mathrm{O}$ autor

Nesta busca identificamos a obra de Gonçalves e Reis (2013).

A Imagem 2 apresenta a busca realizada na Science Direct pelo descritor "GeoGebra".

Imagem 2 - Exemplo de buscas realizadas na ScienceDirect
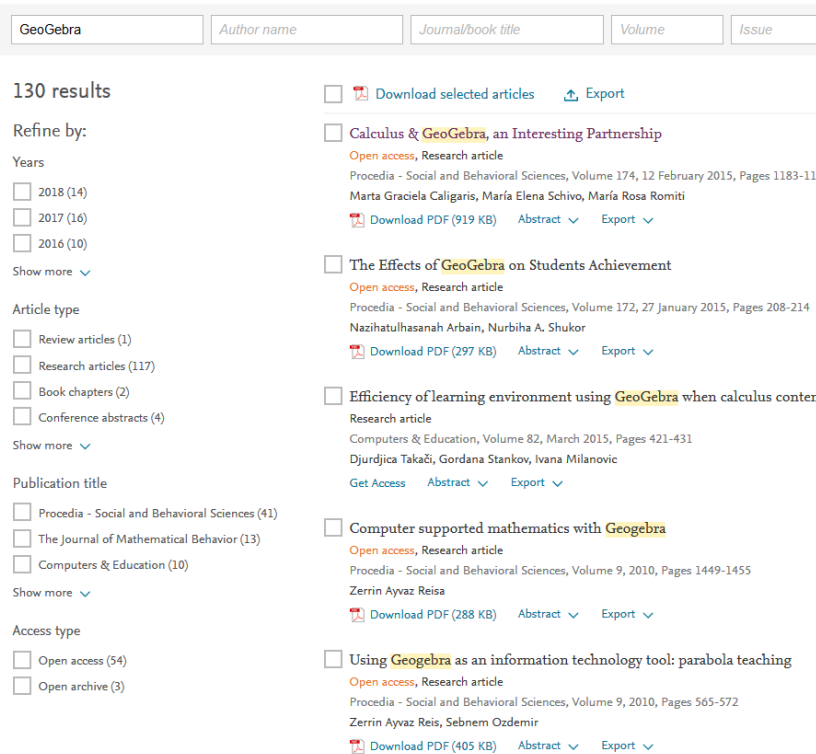

a

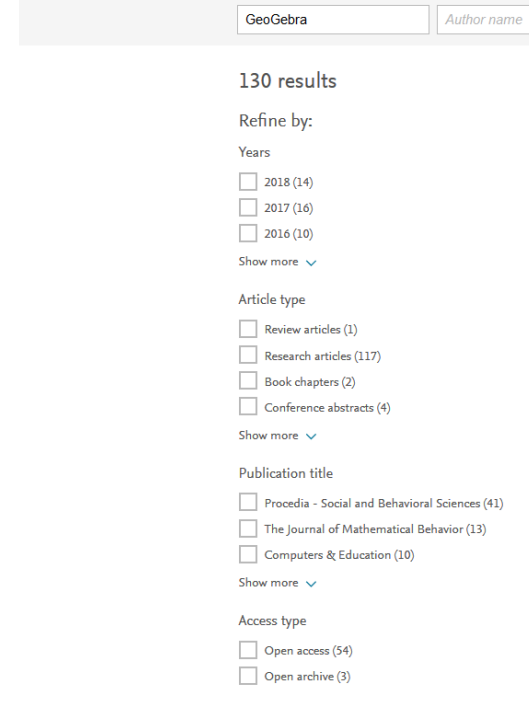

Fonte: $\mathrm{O}$ autor 
Nesta busca identificamos as obras de Calligaris, Schivo e Romoti (2015) e Arango Gaviria e Valencia (2015).

A Imagem 3 apresenta a busca realizada na CAPES pelo descritor "GeoGebra".

Imagem 3 - Exemplo de buscas realizadas na SciELO

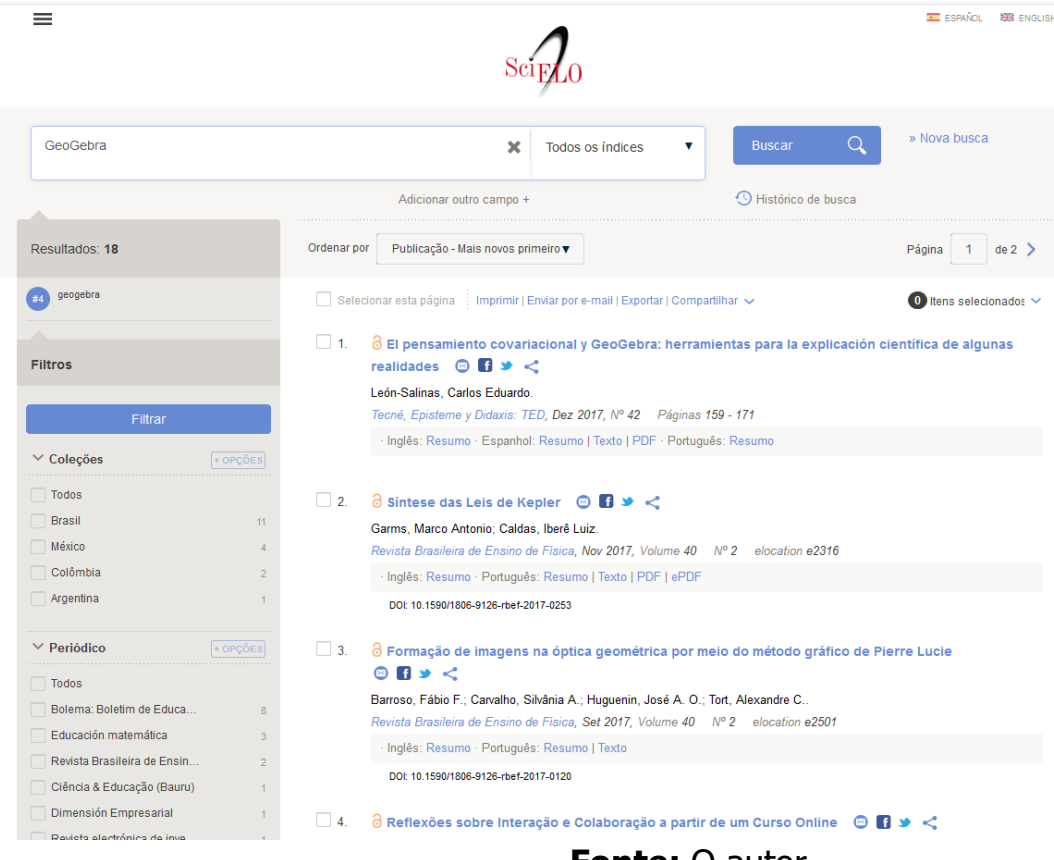

Fonte: $\mathrm{O}$ autor

Nesta busca identificamos a obra de Gonçalves e Reis (2013).

Em seguida, foi realizada nova busca, mas desta vez, suprimindo a palavra Ensino no descritor "Ensino de Cálculo Diferencial e Integral" e interligando com o descritor GeoGebra.

A Imagem 4 apresenta a busca realizada na SciELO pelo descritor "GeoGebra and Cálculo Diferencial e Integral".

Imagem 4 - Exemplo de buscas realizadas na SciELO

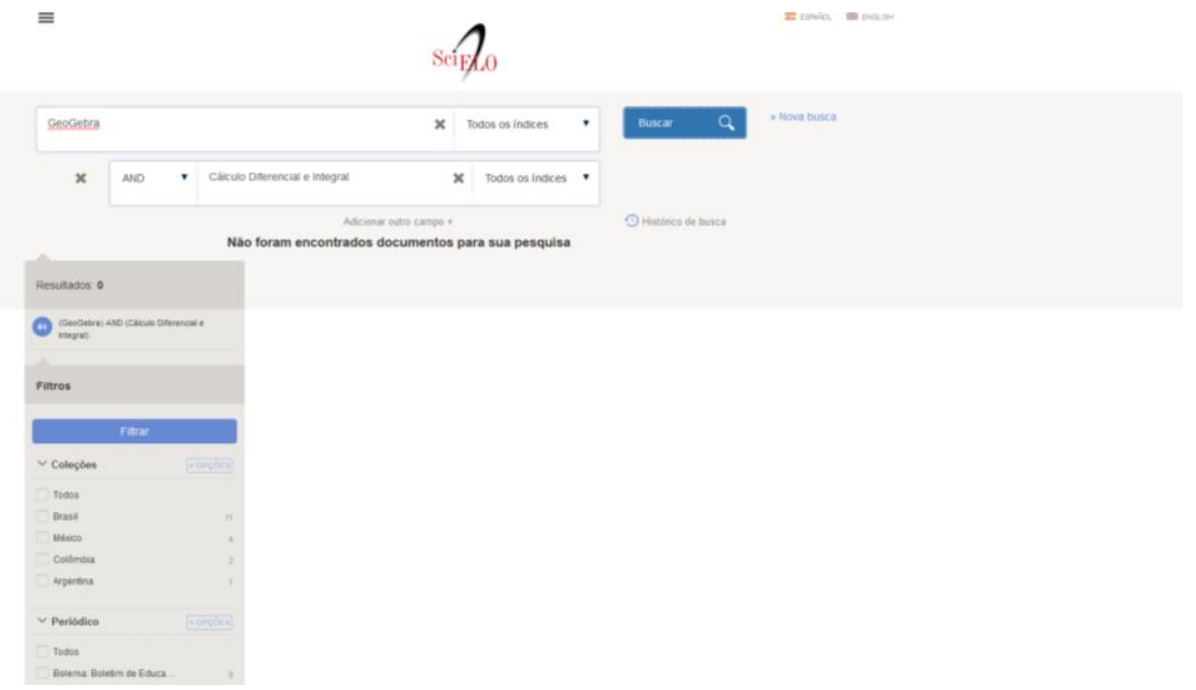

Fonte: O autor 
A Imagem 4 representa uma das buscas onde não obtivemos nenhum resultado.

A Imagem 5 apresenta a busca realizada na CAPES pelo descritor "GeoGebra and Cálculo Diferencial e Integral".

Imagem 5 - Exemplo de buscas realizadas na CAPES

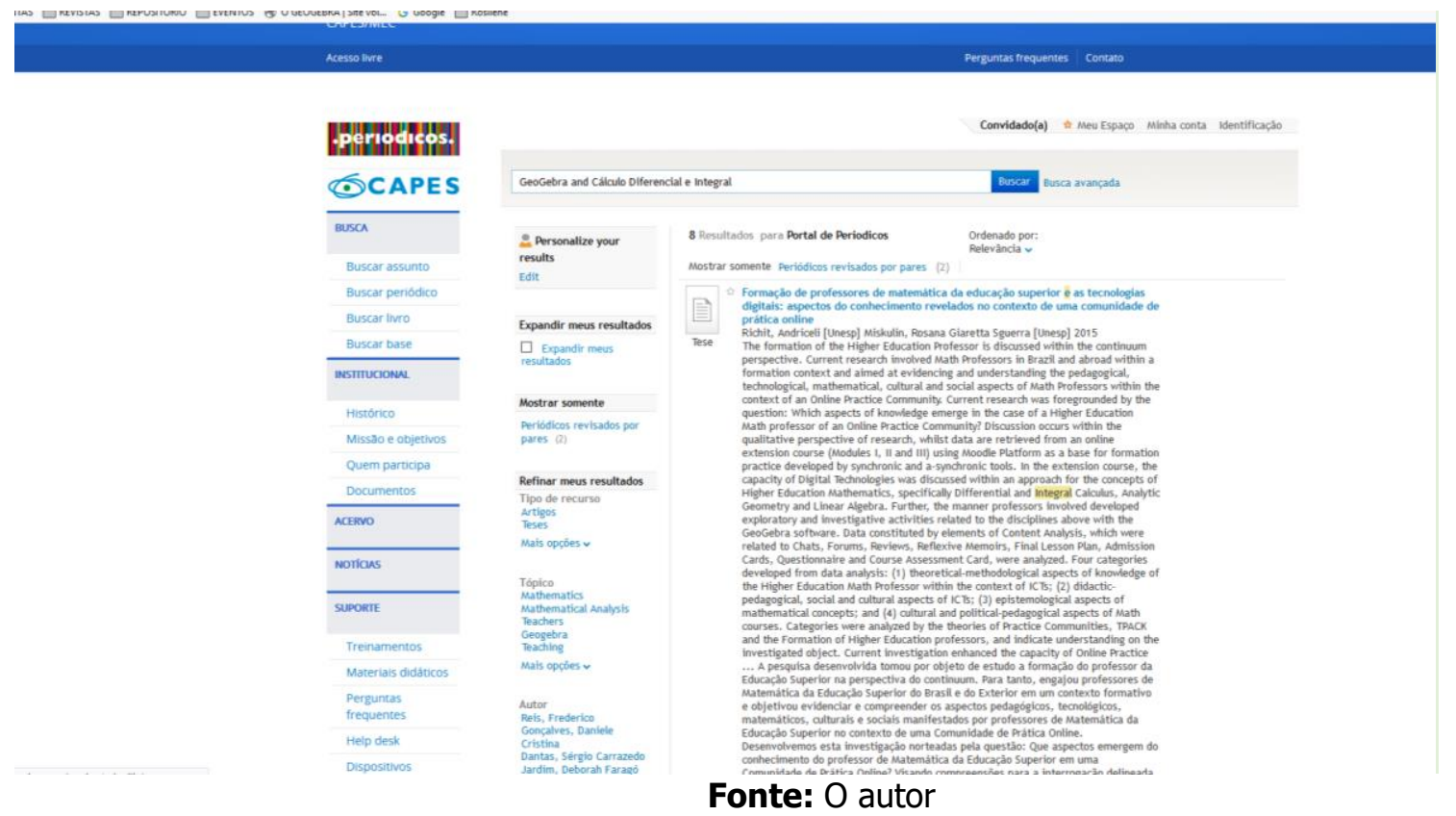

Nesta busca identificamos algumas das obras analisadas.

Por último, a busca que gerou o melhor resultado foi a que considerou os descritores "GeoGebra", "Ensino", e "Cálculo Diferencial e Integral.

A Imagem 6 apresenta a busca realizada na CAPES pelo descritor "GeoGebra and Ensino de Cálculo Diferencial e Integral".

\section{Imagem 6 - Exemplo de buscas realizadas na CAPES}

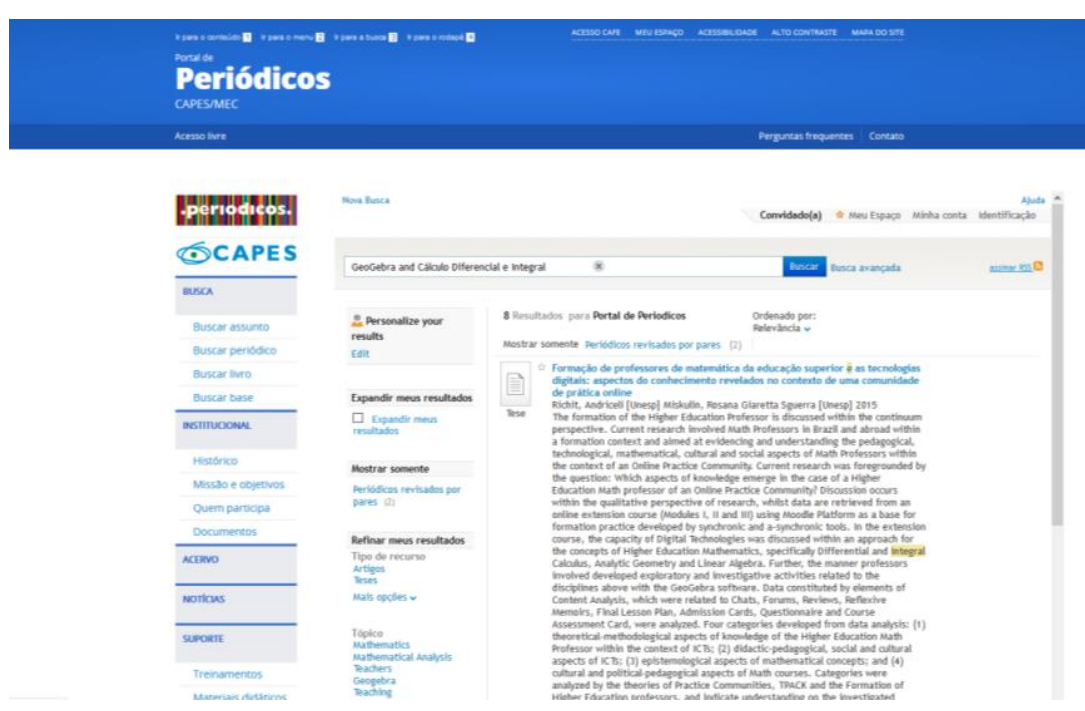

Fonte: $\mathrm{O}$ autor

Nesta busca identificamos a maior parte das obras. 
Ao todo, foram encontradas catorze trabalhos assim distribuídos: onze trabalhos (SIMÕES, 2014; SILVA, 2017; SILVA A, 2017; SANTOS, 2012; PEREIRA, 2016; NUMER e JUSTO, 2015; LIMA, 2017; MARTINS JUNIOR, 2015; CUNHA e LAUDARES, 2017; BIZELLI, BARROSO e FISCARELLI, 2011; ALVES, 2016), nas revistas indexadas na CAPES; um trabalho (GONÇALVES e REIS, 2013) na SciELO; e dois trabalhos (CALLIGARIS, SCHIVO e ROMITI, 2015; ARANGO, GAVIRIA e VALENCIA, 2015) na Science Direct.

\section{SÍNTESE DO MAPEAMENTO}

Ao longo deste trabalho procuramos identificar o que se têm publicado sobre o uso de GeoGebra voltado ao ensino de CDI. Para facilitar na referência das publicações analisadas, elaboramos o Quadro 1 vinculando cada obra a uma letra (maiúscula).

Quadro 1 - Relação das obras (e seus respectivos autores) selecionadas para análise com os respectivos identificadores (letras do alfabeto).

\begin{tabular}{|c|c|c|}
\hline Obras & Autores & Identificador \\
\hline $\begin{array}{l}\text { O comportamento de funções com o estudo de Derivadas por } \\
\text { sequências didáticas em objeto de aprendizagem }\end{array}$ & $\begin{array}{c}\text { Cunha e Laudares } \\
\text { (2017) }\end{array}$ & A \\
\hline $\begin{array}{l}\text { A modalidade EAD semipresencial e a disciplina de Cálculo } \\
\text { Diferencial e Integral }\end{array}$ & Silva (2017) & B \\
\hline $\begin{array}{l}\text { Análises preliminares e a análise a priori para a noção de } \\
\text { Integrais Dependentes de Parâmetros }\end{array}$ & Alves (2016) & C \\
\hline $\begin{array}{l}\text { Atividades investigativas de aplicações das Derivadas utilizando } \\
\text { o GeoGebra }\end{array}$ & $\begin{array}{c}\text { Gonçalves e Reis } \\
\text { (2013) }\end{array}$ & D \\
\hline Calculando áreas sob gráficos de funções & Simões (2014) & $\mathrm{E}$ \\
\hline $\begin{array}{l}\text { Cálculo e Análise: mapeamento das pesquisas do GT } 04 \text { - } \\
\text { educação matemática no Ensino Superior }\end{array}$ & $\begin{array}{l}\text { Lima, Biachini e } \\
\text { Gomes (2017) }\end{array}$ & $\mathrm{F}$ \\
\hline $\begin{array}{l}\text { Cálculo online: uma nova perspectiva para o ensino de Cálculo } \\
\text { Diferencial e Integral }\end{array}$ & $\begin{array}{l}\text { Bizelli, Barroso e } \\
\text { Fiscarelli (2001) }\end{array}$ & G \\
\hline Calculo \& GeoGebra, uma parceria interessante & $\begin{array}{l}\text { Caligari, Shivo e } \\
\text { Romiti (2015) }\end{array}$ & $\mathrm{H}$ \\
\hline $\begin{array}{l}\text { Ensino de Cálculo Diferencial através de objetos virtuais de } \\
\text { aprendizagem no campo de ciências de gestão }\end{array}$ & $\begin{array}{l}\text { Arango, Gaviria e } \\
\text { Valencia (2015) }\end{array}$ & I \\
\hline $\begin{array}{l}\text { Ensino de Derivadas em Cálculo I: aprendizagem a partir da } \\
\text { visualização com o uso do GeoGebra }\end{array}$ & $\begin{array}{l}\text { Martins Junior } \\
\text { (2015) }\end{array}$ & J \\
\hline $\begin{array}{l}\text { Noção de Limite de funções reais e GeoGebra: um estudo em } \\
\text { epistemologia genética }\end{array}$ & A SILVA (2017) & K \\
\hline $\begin{array}{l}\text { Problemas de máximos e mínimos com o auxílio do software } \\
\text { GeoGebra e conhecimento de Derivada }\end{array}$ & $\begin{array}{l}\text { Numer e Justo } \\
\text { (2015) }\end{array}$ & $\mathrm{L}$ \\
\hline $\begin{array}{l}\text { Tarefas para as aulas de Cálculo Diferencial e Integral } \\
\text { implementados com o GeoGebra no contexto da cinemática }\end{array}$ & Pereira (2016) & M \\
\hline $\begin{array}{l}\text { Um estudo sobre a introdução de conceitos de Cálculo no } \\
\text { Ensino Médio }\end{array}$ & Santos (2012) & $\mathrm{N}$ \\
\hline
\end{tabular}

Fonte: $\mathrm{O}$ autor

Passamos então a, resumidamente, apontar as abordagens utilizadas em cada um dos trabalhos do Quadro 01. Essas abordagens resumem-se aos seguintes temas: Ensino de CDI no Ensino Superior; abordagem de CDI no Ensino Médio; e obras que não contemplam especificamente o tema mais que trazem contribuições importantes.

Abordam o ensino de CDI no ensino superior: Cunha e Laudares (2017), Gonçalves e Reis (2013); Martins Junior (2015); A SILVA (2017); Bizelli, Barroso e Fiscarelli (2001); Silva (2017); Caligaris, Schivo, Romiti (2015); Arango, Gaviria e Valencia (2015) e Alves (2016). 
Cunha e Laudares (2017) discorrem sobre o estudo do comportamento de Funções a partir da aplicação da Derivada, através de objetos de aprendizagem (OA) construídos no GeoGebra e sequências didáticas.

Gonçalves e Reis (2013) apresentam um Produto Educacional (construído no GeoGebra) para alunos do curso de Licenciatura em Matemática, com o objetivo de discutir as mudanças que podem ocorrer em sala de aula, quando da utilização de Tecnologias Informacionais e Comunicacionais na Educação (TICEs), aliadas à Atividades Investigativas - (AI) de aplicação de Derivadas.

Martins Junior (2015) realiza uma pesquisa empírica com quatro Professores Universitários Doutores, com aplicação de atividades guiadas exploratórias utilizando o GeoGebra para o ensino de Funções Reais e Derivadas.

A Silva (2017) procurou responder o seguinte questionamento: "Qual a noção de Limite de Funções que alunos da disciplina CDI, do Curso de Licenciatura em Ciências Naturais, apresentam na interação com objetos de aprendizagem do GeoGebra em ambiente virtual" O trabalho é voltado ao estudo dos processos de construção do conhecimento e dos processos cognitivos resultantes das atividades propostas e do uso de vídeo aulas elaboradas com o GeoGebra.

Bizelli, Barroso e Fiscarelli (2001) realizam um estudo teórico, sobre o uso de um material interativo (constituído de applets, animações, atividades dinâmicas, aplicações, aulas digitais, textos e roteiros, que relacionam o uso de gráficos, fórmulas, diagramas e expressões verbais) para estudo de Cálculo Diferencial e Integral I e II com alunos de cursos de Bacharelado e Licenciatura em Química e outras áreas.

Silva (2016) estuda a oferta de cursos de Cálculo Diferencial e Integral I em uma Universidade, na forma de Ensino a Distância (EAD) e Semipresencial, como reforço à alunos em dependência na disciplina, utilizando-se de applets, vídeo aulas e materiais de apoio.

Caligaris, Schivo, Romiti (2015) apresentam algumas animações construídas no Geogebra (na forma de applets), para servir de instrumentos ao ensino de Cálculo, enfatizando a análise de suas interpretações geométricas, a fim de ajudar os alunos, a lidar com representações simbólicas e gráficas, para auxiliar na aprendizagem das definições relativas a esta disciplina.

O trabalho de Arango, Gaviria e Valencia (2015) é uma pesquisa internacional que discorre sobre o uso de objetos de aprendizagem virtual para o ensino de CDI em um curso de Bacharelado em Gestão de Ciências.

Alves (2016) apresenta um estudo conceitual, uma investigação sobre o ensino de Integrais Dependentes de Parâmetros, no Ensino Superior com o uso do GeoGebra.

Sugerindo o ensino de CDI no Ensino Médio, temos: Simões (2014); Numer e Justo (2015), e Santos (2012).

Simões (2014) propõe uma discussão a respeito do estudo de cálculo de área limitada por curvas de gráficos de funções, a partir da abordagem do método da Soma de Riemman.

Numer e Justo (2015) apresentam o GeoGebra como ferramenta para criar applets para abordar o estudo de Máximos e Mínimos de Funções com a utilização de Derivadas com alunos do Ensino Médio. 
E Santos (2012), discorre sobre o uso de sequências didáticas, com atividades investigativas para abordar a introdução do ensino de Cálculo no Ensino Médio, sobretudo, no estudo de Limite e Derivada, e abordagem do estudo de Funções.

Trabalhos que não tratam especificamente do tema, mas que apresentam contribuições importantes são: Lima, Bianchini e Gomes (2017) e Pereira (2016).

Lima, Bianchini e Gomes (2017) apresentam um mapeamento sobre o ensino da disciplina de CDI no Ensino Superior, analisando trabalhos publicados entre 2000 e 2013, no âmbito do GT4 ${ }^{14}$ da SBEM, onde se pode observar que não há trabalhos publicados no período abordando o uso do software GeoGebra para o ensino de CDI no Ensino Superior.

E Pereira (2016) aponta para a possibilidade de se ofertar aulas dinâmicas e contextualizadas, a partir de tarefas direcionadas, de modo a permitir que os alunos explorem os exercícios e encontrem a solução utilizando recursos presentes no site GeoGebraBook ${ }^{15}$.

De acordo com o Quadro 01 podemos observar que, apesar de haver uma diversidade de forma de publicações, a maior parte das pesquisas sobre o tema vem sendo publicadas na forma de Artigos. Por outro lado, a diversidade de formatos de publicações encontradas (Artigos, trabalhos de conclusão de curso de graduação, mestrado e doutorado, entre outras), aponta para a importância deste tema.

\section{ANÁLISE DOS DADOS LEVANTADOS}

Após a análise dos trabalhos selecionados, verificamos que a maior parte deles está voltada para o Ensino Superior, e mais especificamente, para cursos de Graduação na área de Engenharia (ver Quadro 2). É no Ensino Superior que, em geral, são lecionados cursos envolvendo os conceitos de Cálculo Diferencial e Integral, o que justifica o número de trabalhos voltados à sujeitos deste nível de ensino. O fato da maioria dos trabalhos estarem voltados à cursos de Engenharia pode estar relacionado aos altos índices de reprovação nas disciplinas de CDI em cursos de graduação desta área, conforme destaca Martins Junior (2015).

Quadro 2 - Sujeitos das pesquisas analisadas.

\begin{tabular}{|l|c|c|}
\hline Sujeitos da Pesquisa & $\begin{array}{c}\text { Qtd de } \\
\text { trabalhos }\end{array}$ & $\begin{array}{c}\text { Relação de } \\
\text { trabalhos }\end{array}$ \\
\hline Alunos de Licenciatura (universidade) & 4 & CDKF \\
\hline Alunos de outra área (universidade) & 6 & ABGHIM \\
\hline Professores da universidade & 1 & $\mathrm{~J}$ \\
\hline Professores do Ensino Médio & & - \\
\hline Alunos (Ensino Médio) & 3 & ELN \\
\hline
\end{tabular}

Fonte: $\mathrm{O}$ autor

Quanto a natureza destas pesquisas percebemos que não há predominância de uma em detrimento de outra, conforme pode ser visualizado no Quadro 3. Os resultados publicados vêm sendo obtidos por meio de pesquisas empíricas e teóricas, com uma leve vantagem numérica das pesquisas empíricas.

\footnotetext{
${ }^{14}$ Um Grupo de Trabalho de Educação Matemática pertencente à Sociedade Brasileira de Educação Matemática (SBEM), criada no ano 2000 com a finalidade de fomentar investigações sobre o ensino de matemática no nível superior.

${ }^{15} \mathrm{O}$ GeoGebra Book pode ser acessado pelo endereço eletrônico disponível em: < http://tube.geogebra.org/ >
} 
Quadro 3 - Natureza das pesquisas analisadas.

\begin{tabular}{|l|c|c|}
\hline Natureza da pesquisa & Qtd de trabalhos & $\begin{array}{c}\text { Relação de } \\
\text { trabalhos }\end{array}$ \\
\hline Empírica & 8 & ABDJKLMN \\
\hline Teórica & 6 & CEFGHI \\
\hline
\end{tabular}

Fonte: $\mathrm{O}$ autor

No Quadro 4, agrupamos as obras analisadas por finalidade de uso dado ao GeoGebra em processos de ensino de CDI. Os trabalhos de pesquisa analisados apontam para a afirmação de que os OAs ou applets são importantes nos processos de ensino e aprendizagem de CDI, uma vez que, tais mídias foram utilizadas em vários trabalhos como estratégia para conjecturação e exemplificação de conceitos, conforme podemos observar no Quadro 4.

Quadro 4 - Finalidades de uso do GeoGebra observadas nos trabalhos de pesquisas analisados que versam sobre o ensino de CDI com este software.

\begin{tabular}{|l|c|c|}
\hline Finalidades de uso do GeoGebra no ensino de CDI & $\begin{array}{c}\text { Qtd de } \\
\text { trabalhos }\end{array}$ & $\begin{array}{c}\text { Relação } \\
\text { de } \\
\text { trabalhos }\end{array}$ \\
\hline Construir applets, objetos de aprendizagem, animações ${ }^{16}$ & 8 & ABLMGHKI \\
\hline Construir Material Multimídia para Ambiente Educacional & 2 & $\mathrm{GJ}$ \\
\hline Construir um Produto Educacional & 1 & $\mathrm{D}$ \\
\hline Resolver problemas & 2 & $\mathrm{EM}$ \\
\hline Não identificado ou não utiliza & 2 & $\mathrm{CF}$ \\
\hline
\end{tabular}

Fonte: O autor

As informações presentes nos Quadros 4 e 5 nos levam a crer que são as funcionalidades de imprimir dinamismo aos objetos matemáticos e a facilidade de geração de applets (que podem ser considerados objetos de aprendizagem), que mais tem chamado atenção dos pesquisadores para o uso do GeoGebra no ensino de CDI.

Quadro 5 - Estratégias de ensino do CDI empregadas utilizando o software GeoGebra.

\begin{tabular}{|l|c|c|}
\hline Estratégias de ensino & $\begin{array}{c}\text { Qtd de } \\
\text { trabalhos }\end{array}$ & $\begin{array}{c}\text { Relação de } \\
\text { trabalhos }\end{array}$ \\
\hline Explorar atividades no software & 5 & $\mathrm{BCILN}$ \\
\hline $\begin{array}{l}\text { Usar conjuntamente com outras tecnologias digitais em } \\
\text { atividades direcionadas }\end{array}$ & 2 & $\mathrm{BI}$ \\
\hline $\begin{array}{l}\text { Permitir a construção, resolução de problemas e } \\
\text { exploração de conceitos }\end{array}$ & 2 & $\mathrm{IN}$ \\
\hline Sequência Didática & 2 & $\mathrm{NA}$ \\
\hline Situação Problema & 1 & $\mathrm{C}$ \\
\hline Atividades Guiadas Exploratórias & 1 & $\mathrm{~J}$ \\
\hline Atividades Investigativas & 1 & $\mathrm{M}$ \\
\hline Material Complementar & 3 & $\mathrm{DGK}$ \\
\hline $\begin{array}{l}\text { Transitar entre representações simbólica, numérica e } \\
\text { gráfica }\end{array}$ & 1 & $\mathrm{H}$ \\
\hline
\end{tabular}

Fonte: O autor

Percebemos que muitos trabalhos iniciaram o estudo de tópicos de CDI a partir de outros conteúdos, como por exemplo, o trabalho de Numer e Justo (2015) que para abordar o conceito de Derivada, parte do estudo de máximos e mínimos de funções. E poucos estudaram diretamente seu conteúdo como Martins Junior (2015).

\footnotetext{
${ }^{16} \mathrm{Em}$ nosso trabalho, entendemos "animações geradas no GeoGebra, objetos de aprendizagem gerados no GeoGebra e applets, como sendo os mesmos objetos.
} 
De modo geral, nos trabalhos analisados, os autores assumem a exploração do software como fator de modificação e mediação didática. Está explícito nesses trabalhos que a utilização do GeoGebra permite criar situações que favoreçam a interação aluno-máquina, aluno-professor e aluno-aluno, dependendo do modo como as aulas são organizadas. Também está explícito que o GeoGebra proporciona os meios para que os alunos explorem os conceitos envolvidos nas atividades a partir de metodologia da resolução de problemas.

Os Quadros 4 e 5 sugerem que o software permite o uso de diferentes estratégias, que segundo os autores, podem contribuir com a melhora no processo de aprendizagem, e que o recurso da visualização contribui para a ressignificação dos conceitos ligados ao estudo do CDI ao proporcionar equilíbrio entre o processo visual e algébrico.

Os trabalhos de pesquisa analisados apontam para a afirmação de que os OAs ou applets têm se mostrados importantes nos processos de ensino e aprendizagem de CDI, uma vez que, tais mídias foram utilizadas em vários trabalhos como estratégia para conjecturação e exemplificação de conceitos, conforme podemos observar no Quadro 4.

Isso nos leva a crer que são as funcionalidades de imprimir dinamismo aos objetos matemáticos e a facilidade de geração de applets (que podem ser considerados objetos de aprendizagem), que mais tem chamado atenção dos pesquisadores para o uso do GeoGebra no ensino de CDI.

Quadro 6 - Principais resultados das pesquisas

\begin{tabular}{|l|c|}
\hline $\begin{array}{l}\text { Quanto aos resultados esperados ou alcançados na pesquisa, } \\
\text { o uso do GeoGebra se mostra: }\end{array}$ & Qtd de trabalhos \\
\hline Eficiente ${ }^{17}$ & 13 \\
\hline Ineficiente & 0 \\
\hline Não abordou & 1 \\
\hline
\end{tabular}

Fonte: $\mathrm{O}$ autor

O Quadro 6 aponta para o entendimento de que a utilização do software GeoGebra, no ensino de CDI, favorece o aprendizado de seus conceitos, sobretudo, quando direcionado aos objetivos apresentados no Quadro 7 e aliado às frequências de utilização apresentadas no Quadro 8.

No Quadro 7 são apresentados os principais argumentos/razões identificadas nas obras analisadas pelas quais o software GeoGebra é utilizado para o ensino de CDI.

Quadro 7 - Alguns dos objetivos propostos nas pesquisas.

\begin{tabular}{|l|}
\multicolumn{1}{c|}{ Objetivos propostos nas pesquisas } \\
\hline Favorecer interação e descoberta aluno-AO \\
\hline Favorecer descobertas e consequentemente, questionamentos e discussão \\
\hline Visualização de conceitos para melhor compreendê-lo \\
\hline Resolução das atividades \\
\hline
\end{tabular}

Fonte: O autor

Como podemos observar, os objetivos identificados estão centrados no estímulo à interação, na resolução de atividades por meio de questionamentos levantados pelos professores durante a

\footnotetext{
17 Enquadramos aqui tanto as pesquisas que apenas sugerem o uso do software GeoGebra, por entendermos que apresentam potencialidades aos processos de ensino aprendizagem, quanto as que comprovaram eficácia do software para o ensino de CDI.
} 
manipulação de applets pelos alunos, e na proposta de visualização dos objetos matemáticos com o GeoGebra. Logo, podemos deduzir, com base nestes resultados, que as principais estratégias de uso do GeoGebra para o ensino de CDI estão centradas nas possibilidades de dinamismos dos objetos matemáticos proporcionadas pelo software.

No Quadro 8 são apresentadas as principais sugestões de uso do GeoGebra no ensino de CDI, identificadas nos trabalhos analisados.

Quadro 8 - Lista de sugestões de uso do GeoGebra identificadas nas publicações analisadas.

\begin{tabular}{|l|}
\hline \multicolumn{1}{|c|}{ Sugestões de uso do GeoGebra } \\
\hline Contínuo em sala de aula \\
\hline Esporádico em sala de aula \\
\hline Transitar entre representações numérica, algébrica e geométrica \\
\hline Contínuo no laboratório da escola \\
\hline Esporádico no laboratório da escola \\
\hline Para explicação do conteúdo \\
\hline Para explorar conceitos após explicação do conteúdo \\
\hline Para investigação em atividades \\
\hline Para resolução de atividades \\
\hline Para verificação de resultados \\
\hline
\end{tabular}

Fonte: $\mathrm{O}$ autor

Analisando, em conjunto, os resultados apresentados nos Quadros 5, 7 e 8, é possível perceber que não há um padrão adotado para a utilização do GeoGebra no ensino de CDI, ou seja, não há um design de curso, ou ainda, não há reconhecimento, entre os pesquisadores da área, da existência de um modelo de proposta de uso do GeoGebra para o ensino de Cálculo Diferencial e Integral. Embora sabemos que a existência de tal modelo "milagroso" é impossível, percebe-se nos resultados que não há convergência das propostas, a não ser pelo caráter dinâmico do software.

Quadro 9 - Classificação das pesquisas quanto à apresentação de dificuldades e/ou contribuições nos processos de ensino de Cálculo Diferencial e Integral.

\begin{tabular}{|l|c|c|}
\hline \multicolumn{1}{|c|}{ Descrição } & $\begin{array}{c}\text { Qtd de } \\
\text { trabalhos }\end{array}$ & $\begin{array}{c}\text { Identificação } \\
\text { dos trabalhos }\end{array}$ \\
\hline $\begin{array}{l}\text { Não apresentaram nem as dificuldades e nem as } \\
\text { contribuições }\end{array}$ & 3 & $\mathrm{~A}, \mathrm{~F}, \mathrm{G}$ \\
\hline Apresentam somente contribuições & 5 & $\mathrm{C}, \mathrm{E}, \mathrm{H}, \mathrm{I}, \mathrm{L}$ \\
\hline Apresentam somente dificuldades & 1 & $\mathrm{M}$ \\
\hline Apresentam dificuldades e contribuições & 5 & $\mathrm{~B}, \mathrm{D}, \mathrm{J}, \mathrm{K}, \mathrm{N}$ \\
\hline
\end{tabular}

Fonte: $\mathrm{O}$ autor

Verificamos no Quadro 9 que muitas das pesquisas discorreram sobre dificuldades no ensino de Cálculo. Percebemos durante as leituras, que muitas obras apontam dificuldades presentes na literatura atual, referentes ao ensino de CDI, e igualmente, sugestões de melhorias em seu ensino.

\section{PRINCIPAIS DIFICULDADES E SUGESTÕES PARA O ENSINO DE CÁLCULO DIFERENCIAL E INTEGRAL IDENTIFICADAS DURANTE O MAPEAMENTO}

Ao invés de tentarmos tabular as dificuldades relacionadas ao ensino do CDI, bem como as principais sugestões/recomendações de estratégias de ensino para o enfrentamento destas dificuldades, optamos por apresentar, de forma, sucinta o que apontam os autores das obras analisadas neste 
trabalho. Assim, acreditamos ser possível exprimir com fidedignidade as contribuições de cada autor sobre este tema.

Silva (2017), aponta como desafio para o ensino de Cálculo: a falta de formação básica dos alunos; a transição no raciocínio que o aluno se obriga a realizar para dar conta dos conceitos novos abordados na universidade; a transição entre o raciocínio algébrico para o analítico; e a dificuldades na relação aluno-professor.

O autor Sugere que o professor conheça melhor sua própria realidade e prática pedagógica e faça uma reflexão sobre ela e sobre a utilização de tecnologias digitais como: Slides, tutoriais, notas de aulas, atividades práticas online, softwares (como o GeoGebra), vídeo aulas, whatsapp, facebook, Ambiente Virtual de Aprendizagem e outros, para o ensino do CDI, por entender que estes recursos podem contribuir nos processos de de ensino e aprendizagem.

Alves (2016), faz uma discussão sobre o tema, mas não se aprofunda nas possíveis dificuldades. Para melhorar a qualidade do ensino desta disciplina, o autor sugere o uso da abordagem metodológica de Transição Interna do Cálculo, que segundo Tall (1985), é uma espécie de transição entre diferentes conceitos ou de conceitos que se complementam. Sugere também o uso do software GeoGebra para viabilizar a transmissão didática do conteúdo no processo de ensino, para a construção de atividades, e exploração em sala de maneira corriqueira.

Gonçalves e Reis (2013), assumem a condição de que os professores deveriam saber onde e para que o conhecimento de CDI se faz importante. Para os autores, a dificuldade no ensino de Cálculo estaria relacionada ao processo de ensino, e, possivelmente, na dificuldade de entendimento do conceito de Limite e na falta de uma abordagem que possibilite o aluno aplicar este conhecimento. Os autores sugerem uma mudança de postura por parte dos professores como uma alternativa para enfrentar esta problemática.

No trabalho de Simões (2014), não são apontadas as razões das dificuldades no ensino de CDI, mas descreve, em suas considerações, a necessidade de abordagem dos conceitos do Cálculo com alunos do Ensino Médio, para que estes cheguem ao Ensino Superior com melhores condições de lidarem com os conceitos do CDI.

Caligaris, Schivo, Romiti (2015), também não abordam as causas, mas afirmam que a utilização de applets, gerados no GeoGebra, e as situações de ensino que nele podem ser geradas, se apresentam como uma metodologia de ensino mais significativa do que a abordagem tradicional de ensino desta disciplina.

Arango, Gaviria e Valencia (2015), abordam os problemas do ensino de CDI mas sem se aterem nas possíveis causas. Sugerem o uso de $\mathrm{VLO}^{18}$ e vídeo aulas na dinâmica da sala de aula, como possibilidades de contribuição nos processos de aprendizagem da disciplina. Entendem que a utilização desses recursos, de forma conjunta, pode melhorar a assimilação do conhecimento teórico em sala de aula, uma vez que, diferentes processos dinâmicos, algorítmicos e heurísticos podem ser empregados utilizando estes recursos.

\footnotetext{
18 Do inglês "Virtual Learning Objects"(VLO) ou Objetos de Aprendizagem Virtual.
} 
O trabalho de Martins Junior (2015) foi o que mais abordou as dificuldades relativas ao ensino do Cálculo, entre elas o autor destaca:

"saber se as estratégias usadas com os alunos estão realmente proporcionando aprendizagem; falta de conhecimentos específicos e pedagógicos dos conteúdos pelos professores para saber se os seus recursos utilizados estão sendo eficientes durante o ensino; enorme quantidade de alunos nas turmas de Cálculo o que, na maioria das vezes, dificulta o acesso ao laboratório; ementa da disciplina de Cálculo que, geralmente, é incompatível com a carga horária disponível; dificuldades na transição de conteúdos elementares para conteúdos avançados; dificuldades epistemológicas que estes conteúdos possuem tanto para o ensino como para a aprendizagem" (MARTINS JUNIOR, 2015, p. 116).

O autor sugere como proposta de ensino a utilização de applets ,criados no GeoGebra, para desenvolver atividades exploratórias com alunos no laboratório de informática, e a exploração de OA voltados para Ensino Superior, como suporte ao ensino.

Silva A (2017) discorre sobre o assunto e alega como causas: Dificuldades dos professores em associar teoria e prática; falhas na estrutura da disciplina; e falta de base conceitual pelos alunos, especialmente sobre os conceitos de Limite e Funções. O autor vê na possibilidade de conhecer os processos cognitivos do aluno, a possibilidade de orientar seu desenvolvimento e oportunizar sua aprendizagem em um contexto de ensino.

Numer e Justo (2015) não apontam possíveis dificuldades no ensino de Cálculo, más sugerem a inclusão desta disciplina ao currículo do Ensino Médio. Santos (2012) aponta que as dificuldades no ensino de CDI muitas vezes têm haver com: A falta de interesse com a formação dos alunos ainda no ensino básico; de motivação dos alunos; a falta de associação dos conceitos estudados com sua aplicação; e na dificuldade de se entender os conceitos da disciplina, decorrentes da metodologia utilizada pelos professores. Sugere como proposta a abordagem do Cálculo para alunos do Ensino Médio.

Pereira (2016), Cunha e Laudares (2017), Bizelli, Barroso e Fiscarelli (2001), Lima, Bianchini e Gomes (2017) não desenvolveram argumentos sobre o tema.

Em síntese, percebemos que as principais dificuldades apontadas nas pesquisas giram em torno da falta de bagagem conceitual dos alunos e das metodologias de ensino comumente utilizadas.

As principais propostas de enfrentamento estão relacionadas à mudança de postura dos professores (aspectos metodológicos) e a incorporação de tecnologias digitais, sobretudo do GeoGebra, em especial de applets (construídos neste software), para favorecer a visualização geométrica dos conceitos e a transição entre diferentes representações matemática do objeto em estudo.

\section{CONSIDERAÇÕES}

De acordo com Lima, Bianchini, Gomes (2015), Martins Junior (2015), Gonçalves e Reis (2013), há tempo o ensino de CDI vem sendo objeto de estudo em diversas pesquisas, tanto em âmbito nacional como internacional. No entanto, sua abordagem utilizando o GeoGebra, parece-nos ainda tímida.

Com o intuito de contribuir com a temática, realizamos um mapeamento sobre as pesquisas publicadas que abordam o "Ensino de Cálculo Diferencial e Integral com o uso do software Geogebra. 
Para nortear a realização do trabalho, levantamos os seguintes questionamentos: A pesquisa é nacional ou internacional? Quais são seus sujeitos? A pesquisa é empírica ou teórica? Discorre sobre dificuldades no ensino de Cálculo? Sugere propostas de atividades que possam contribuir com seu enfrentamento? E quanto à abordagem de uso do GeoGebra nestes trabalhos, buscamos verificar se estas destinam-se a: (1) realizar explicação de um dado conteúdo? (2) explorar conceitos a partir de construções realizadas pelos sujeitos de pesquisa? e/ou (3) construir objetos digitais de aprendizagem?

Ao longo do trabalho já apresentamos respostas a estes questionamentos, contudo, julgamos necessário enfatizar ainda os resultados obtidos que consideramos serem mais relevantes para contribuir com a temática.

Ao procurarmos identificar as razões apontadas pelos pesquisadores, para a dificuldade no ensino de Cálculo e suas sugestões de enfrentamento, identificamos, dentre as causas mais apontadas, os seguintes argumentos: (1) a falta de base dos alunos (SILVA, 2017); (2) falhas nas metodologias de ensino dos professores (GONÇALVES e REIS, 2013; SILVA, 2017); (3) falha da estrutura curricular dos cursos (MARTINS JUNIOR, 2015); e (4) a complexidade dos conceitos (MARTINS JUNIOR, 2015; SILVA A, 2017).

Como possíveis contribuições para seu tratamento, destacam-se: (1) a utilização de novas abordagens metodológicas (GONÇALVES e REIS, 2013); (2) melhor preparação do professor quanto ao conteúdo ensinado e suas práticas pedagógicas (SILVA, 2017); (3) o uso intensivo de TICs (ALVES, 2016) e do GeoGebra (CALIGARIS, SCHIVO e ROMITI, 2015); e (4) introdução do ensino de CDI no Ensino Médio (SIMÕES, 2014).

Quanto às abordagens de uso do GeoGebra para o ensino de CDI, destacam-se: (1) aulas diferenciadas (ARANGO, GAVIRIA e VALENCIA, 2015); (2) aulas guiadas com uso de applets (SANTOS, 2012); (3) atividades exploratórias a partir da construção do objeto pelos alunos (MARTINS JUNIOR, 2015); e (4) explicação de conceitos (GONÇALVES e REIS, 2013). Percebe-se não haver consenso entre as formas de se utilizar o GeoGebra.

Com relação às modalidades de curso nas quais os trabalhos de pesquisas analisados haviam sido direcionados, encontramos trabalhos voltados à modalidade: Presencial (NUMER e JUSTO, 2015; SANTOS, 2012); Semipresencial (SILVA, 2017) e Online (GONÇALVES e REIS, 2013).

Quanto ao uso do Geogebra em cursos de CDI, identificamos: o uso cotidiano pelo professor em sala de aula (NUMER e JUSTO, 2015; SANTOS, 2012); em momentos específicos do curso ou como complementação do curso (SILVA A, 2017; BIZELLI, BARROSO e FISCARELLI, 2001); e para reforço pedagógico à alunos com dificuldades na disciplina (SILVA A, 2017).

Percebemos também a utilização do GeoGebra associada com outros recursos, tais como: aplicativos de redes sociais, ambientes virtuais, videoaulas, animações e outros (MARTINS JUNIOR, 2015; GONÇALVES e REIS, 2013; SANTOS, 2012; BIZELLI, BARROSO e FISCARELLI, 2001).

Procurando apontar os argumentos pelos quais o software é utilizado, identificamos que, em geral, os autores assumem a ideia de que, ao explorar o GeoGebra, este se torna um fator de modificação à compreensão dos conceitos e uma importante ferramenta de mediação didática. Os autores também percebem no software a presença de recursos tecnológicos com potencial para contribuir com a 
melhora do processo de ensino do CDI, em especial, os recursos de visualização gráfica e animação, os quais possibilitam a ressignificação dos conceitos relacionados a esta disciplina, e proporcionam o equilíbrio entre o processo visual e algébrico.

Quanto a existência de um designer de curso, toda essa diversidade de métodos, objetivos e propostas de utilização do software Geogebra, nos leva a crer que ainda não há um designer formatado, validado e amplamente utilizado para o ensino de Cálculo Diferencial e Integral.

Por fim, com base nos dados levantados com este mapeamento, chegamos ao entendimento que 0 uso do GeoGebra é significativo nos processos de ensino e aprendizagem dos conceitos relacionados ao Cálculo Diferencial e Integral, e seu uso é recomendável como recurso didático e metodológico em sala de aula.

Dando-nos a percepção de ser viável e importante seu uso para o ensino no fazer pedagógico do professor em sala de aula.

\section{REFERÊNCIAS}

ABRAGNANDO, N. Dicionário de filosofia. 2 ed. São Paulo: Mestre Jou, 1962.

ALVES, Francisco Régis Vieira. Análises preliminares e a análise a priori para a noção de Integrais Dependentes de Parâmetros. VIDYA, Santa Maria, vol: 36, n.1, pp: 111-133 06/2016. Disponível em: < www.periodicos.unifra.br/index.php/VIDYA/article/view/592> acesso 02/02/18.

ARANGO, Juan; GAVIRIA, Diana; VALENCIA, Alerrandro. Differential Calculus Teaching Throgh Learning Objects in the Field of Management Sciences. Procedia - Social and Behavioral Sciences vol: 176, n. 1 pp: 412-418 02/2015. Disponível em: $<$ www.sciencedirect.com/science/article/pii/S1877042815005273 > acesso 02/02/18

BIZELLI, Maria Helena S. S; BARROSO, Bizelli Sidineia; FISCARELLI, Silvio Henrique.Cálculo online: uma nova perspectiva para o ensino de Cálculo Diferencial e Integral. CONGRESSO ESTADUAL PAULISTA SOBRE FORMAÇÃO DE EDUCADORES, 11.; CONGRESSO NACIONAL DE FORMAÇÃO DE PROFESSORES, 1., 2011, Águas de Lindóia. Por uma política nacional de formação de professores. São Paulo: UNESP; PROGRAD. p. 6669-6680 2011 Disponível em: $<$ http://hdl.handle.net/11449/139612 > acesso 02/02/18.

BRASIL; Coordenação de Aperfeiçoamento de Pessoal de Nível Superior CAPES. MEC. Brasil. Disponível em:< http://portal.metodista.br/portal-capes > Acesso em: 06/02/2018.

BRASIL. Scientific Electronic Libray Online - Brasil. Base de Dados disponível em: < http://www.scielo.br/?Ing=pt > Acesso em: 06/02/2018.

CALIGARIS, Marta Graziela; SCHIVO, Maria Elena; ROMITI, Maria Rosa. Calculus \& Amp, GeoGebra, na interesting partnership. Procedia - Social and Behavioral Sciences, vol: 174 n. 1, pp: 1183-1188, 02/2015. Disponível em: <www.sciencedirect.com/science/article/pii/S1877042815007879> acesso 02/02/18

CUNHA, Luiz Gonzaga Alves da; LAUDARES, João Bosco. 0 comportamento de funções com o estudo de derivadas por sequências didáticas em objeto de aprendizagem. VIDYA, Santa Maria, vol: 37, n. 2, pp: 397-416, 07/2017 Disponível em: <www.periodicos.unifra.br/index.php/VIDYA/article/view/2102> acesso 02/02/18. 
Gale A Cengage Company; Cengage Learning, Estados Unidos da América. Base de dados disponível em:< https://www.gale.com/uk > Acesso em 06/02/2018.

GARNICA, Antonio Vicente Maraffioti.; PEREIRA, Maria Eliza. A pesquisa em Educação Matemática no Estado de São Paulo: um possível perfil. Boletim de Educação Matemática (BOLEMA), Rio Claro, v. 11, n. 12, p. 59-74, ??/1997

GONÇALVES, W. V. O Transitar entre a Matemática do Matemático, a Matemática da Escola e a Matemática do GeoGebra: Um Estudo de como Professores de Matemática lidam com as possibilidades e limitações do GeoGebra. Tese (Doutorado) - Universidade Estadual Paulista. Faculdade de Ciências, Bauru, 2016. 240 f. : il.

GONÇALVES, Daniele Cristina; REIS, Frederico da Silva. Atividades investigativas de aplicações das derivadas utilizando o GeoGebra. Bolema: Boletim de Educação Matemática, Rio Claro, vol: 27 n. $46 \quad$ pp: 417-432. 10/203. Disponível em: $<$ www.scielo.br/scielo.php?script=sci arttext\&pid=S0103636X2013000300006\&lng=pt\&tlng=pt $>$ acesso 02/02/18.

MARTINS JUNIOR, Jose Cirqueira. Ensino de Derivadas em Cálculo I: aprendizagem a partir da visualização com o uso do GeoGebra. Ouro Preto, UFOP, 2015. Dissertação de Mestrado Profissional junto a Universidade Federal de Ouro Preto, Instituto de Ciências Exatas e biológicas, Departamento de Matemática, 2015.

LIMA, Gabriel Loureiro de; BIANCHINI, Barbara Lutaif; GOMES, Eloiza. Cálculo e Análise: Mapeamento das pesquisas do GT 04- Educação Matemática no Ensino Superior. VIDYA, Santa Maria, vol: 37 (2) pp: 317-334, 2017 Disponível em: <www.periodicos.unifra.br/index.php/VIDYA/article/view/2009> acesso 02/02/18.

NUMER, Francine Mirele Numer; JUSTO, Dagoberto Adriano Rizzotto. Problemas de máximos e mínimos com auxílio do software GeoGebra e conhecimentos de Derivada. Porto Alegre, UFRGS, 2015. Trabalho de Conclusão de Especialização, junto a Universidade Federal do Rio Grande do Sul. Instituto de Matemática. Matemática, Mídias Digitais e Didática: tripé para formação do professor de matemática, 2015. Disponível em: <http://www.lume.ufrgs.br/handle/10183/134098> acesso 02/02/18.

PALANCH, Wagner Barbosa de Lima. Mapeamento de pesquisas sobre currículos de Matemática na Educação Básica Brasileira. São Paulo, PUC, 2016. Tese Doutorado em Educação Matemática - Programa de Estudos Pós-Graduados em Educação Matemática, Pontifícia Universidade Católica de São Paulo, 2016. (1987 a 2012). 2016. 297 f.

PEREIRA, Patrícia Aparecida Caldana. Tarefas para aulas de Cálculo Diferencial e Integral implementados com o GeoGebra no contexto da cinemática. Londrina, UTFPR, 2016. Trabalho de Conclusão de Curso junto a Universidade Tecnológica Federal do Paraná, Departamento Acadêmico de Ciências humanas, $2016 . \quad$ Disponível em: $<$ http://repositorio.roca.utfpr.edu.br/jspui/handle/1/7412 > acesso 02/02/18

SANTOS, Marcelo de Souza. Um estudo sobre a introdução de conceitos de cálculo no Ensino Médio. Porto Alegre, UFRGS, 2012. Trabalho de Conclusão de Curso junto a Universidade Federal do Rio Grande do Sul, Instituto de Matemática, Departamento de Matemática Pura e Aplicada, 2012.

SILVA, Armando Paulo da. A modalidade EAD Semipresencial e a disciplina de Cálculo Diferencial e Integral. Bauru, UNESP, 2017. Tese de doutorado junto a Universidade Estadual 
Paulista, Programa de Pós-Graduação em Educação para Ciências, da Faculdade de Ciências, Campus de Bauru, 2017.

SILVA, Antônio José da. Noção de Limites de funções reais e GeoGebra: um estudo em Epistemologia Genética. Porto Alegre, UFRGS, 207. Tese de Doutorado junto a Universidade Federal do Rio Grande do Sul, Centro Interdisciplinar de novas tecnologias na educação, Programa de Pós-Graduação em Informática na Educação, 2017. Disponível em:

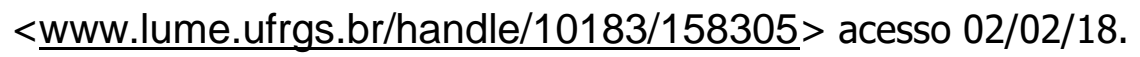

SIMÕES, Alexandre Calligaris. Calculando áreas sob gráficos de funções. Rio Claro. UNESP, 2014. Dissertação de Mestrado apresentada junto a Universidade Estadual Paulista Júlio de Mesquita Filho, Instituto de Geociências e Ciências Exatas, Campus de Rio Claro, 2014.

Science Direct. Editora Elsevier Brasil. Base de Dados Science Direct disponível em: <https://www.sciencedirect.com/> Acesso em: 06/02/2018.

Scopus; Editora Elsevier Brasil. Base de Dados Scopus disponível em: <https://www.elsevier.com/americalatina/pt-br/scopus > Acesso em: 06/02/2018.

Web Of Science; Clarivate Analytics, Canadá. Base de Dados Web Of Science disponível em: <https://webofknowledge.com/ > Acesso em: 06/02/2018 